\title{
RARE COMPLICATION OF A COMMONLY PERFORMED PROCEDURE: THE PEG ALLERGY
}

\author{
Shenthil Prabhu Murugesan', Anbalagan Pichaimuthu², Senthil Kumar Perumal ${ }^{3}$, Jeswanth Satyanesan ${ }^{4}$, Ravichandran Palaniappan $^{5}$
}

1Post Graduate, Department of Surgical Gastroenterology, Government Stanley Medical College.

${ }^{2}$ Assistant Professor, Department of Surgical Gastroenterology, Government Stanley Medical College.

${ }^{3}$ Assistant Professor, Department of Surgical Gastroenterology, Government Stanley Medical College.

${ }^{4}$ Associate Professor, Department of Surgical Gastroenterology, Government Stanley Medical College.

5Professor, Department of Surgical Gastroenterology, Government Stanley Medical College.

ABSTRACT: CASE REPORT: Anaphylaxis to oral PEG.

BACKGROUND: Mechanical Bowel preparation (MBP) is commonly practised for patients prepared for major abdominal surgeries and colonoscopies. Patients with periampullary tumour planned for pancreaticoduodenectomy had their bowel prepared the day before surgery. The importance of this case report is to highlight the rarest of complications of a seemingly innocuous and commonly performed procedure

CASE REPORT: A 47 year old female, a case of periampullary carcinoma with no comorbid illness, was planned for pancreaticoduodenectomy. She had features of obstructive jaundice with cholangitis for which endoscpoc biliary stenting was done. Following consumption of oral PEG solution the day before surgery, patient developed rash, hypotension and breathing difficulty which necessitated ICU admission and postponement of proposed surgery.

CONCLUSION: Our case highlights a rare but potentially life threatening adverse reaction to PEG solutions. A greater awareness of this rare complication is essential for the treating physicians to effectively manage this entity.

KEYWORDS: Anaphylaxis to Peg , Peg and Side Effects, Reactions to Oral Peg.

HOW TO CITE THIS ARTICLE: Shenthil Prabhu Murugesan, Anbalagan Pichaimuthu, Senthil Kumar Perumal, Jeswanth Satyanesan, Ravichandran Palaniappan. "Rare Complication of a Commonly Performed Procedure: The Peg Allergy". Journal of Evolution of Medical and Dental Sciences 2015; Vol. 4, Issue 89, November 05; Page: 15496-15497, DOI: 10.14260/jemds/2015/2216.

INTRODUCTION: Mechanical Bowel preparation (MBP) is commonly practised for patients prepared for major abdominal surgeries and colonoscopies. patients with periampullary tumour planned for pancreaticoduodenectomy had their bowel prepared the day before surgery. PEG solutions are commonly used for mechanical bowel preparations and allergic reactions to PEG an osmotic laxative is rare entity.

CASE REPORT: Herein we have a 47 year old female, a case of Periampullary tumour admitted at our Hepato Pancreatico Biliary Unit who had been planned for Pancreaticoduodenectomy. She had symptoms of pruritus, jaundice with high coloured urine and clay coloured stools. patient had no comorbid illness. Following admission patient developed cholangitis for which patient underwent Endoscopic Biliary stenting. Following Biliary stenting her symptoms subsided and parenteral antibiotics started for cholangitis were stopped and patient was planned for Pancreaticoduodenectomy. She was advised to consume PEG solution the day before surgery. She was not getting any parenteral or oral durgs before consuming PEG solution.

Financial or Other, Competing Interest: None.

Submission 06-10-2015, Peer Review 07-10-2015,

Acceptance 23-10-2015, Published 05-11-2015.

Corresponding Author:

Senthil Prabhu Murugesan,

Block Number 601, New SGE Block,

Govt. Stanley Medical College,

Old Jail Road, Royapuram,

Chennai-600001, Tamilnadu.

E-mail: mshenthil@rediffmail.com

DOI: $10.14260 /$ jemds $/ 2015 / 2216$.
Following ingestion of 4 ounces of PEG solution (PEG powder diluted in $4 \mathrm{~L}$ of water) within half an hour of ingestion patient developed rash all over the body, hypotension, angioedema and breathing difficulty. PEG solution contained sodium Bicarbonate $3.37 \mathrm{gm}$, Sodium Chloride 2.93gm, Potassium Chloride 1.484gm, Sodium Sulphate 11.36gm and PEG electrolyte 118gm. The PEG powder that was used had its expiry in march 2017. She was treated with Injection Chlorpheniramine $10 \mathrm{mg}$ slow IV and injection hydrocortisone $200 \mathrm{mg}$ IV. she was also given fluid challenge with $750 \mathrm{ml}$ of crystalloid solution. Her blood pressure gradually improved and her symptoms subsided.

She was observed in ICU for 24 hours due to respiratory compromise. Subsequently planned elective surgery was postponed to a later date. The batch number of the PEG powder was noted and none of the other patients consuming PEG powder of the same batch during the same period developed any adverse reaction.

DISCUSSION: PEG solutions are commonly used for MBP before major abdominal surgeries and colonoscopies. PEG, a Macrogol derivative, is a polymer of ethylene glycol, used as laxative (Higher weight variants) or excipient (Lower weight variants). Generally PEG solutions are well tolerated and Electrolyte disturbances following PEG is common while allergic reactions are rare yet can be potentially life threatening making it important for the treating physician to know this complication. Adverse reactions of PEG include nausea, vomiting, diarrhea, stomach cramps, thirst, lacrimation, sweating, fever, tachycardia, hypertension, renal dysfunction, edema, shortness of breath, headache, dizziness, anxiety, weakness, twitching and muscle hypertonicity. 
Contraindications for PEG administration include Hypernatremia, hyperhydration, the threat of pulmonary edema and cerebral edema.

So far 6 cases has been reported. Assal and Watson.[1] reported a case with perioral edema and Stallman mantan. ${ }^{[2]}$ reported a case presenting with rash and Schuman and Balsam. ${ }^{[3]}$ reported case presenting with hypotension and flushing. Brullet et al..[4] and Haneda et al.[5] reported a case presenting with urticaria. Julia et al had presented a case with biphasic presentation of allergic symptoms.[6] Stefano Pizzimenti et al reported a case presenting with mild urticarial.[7] Belsey et al published significant events in 22 patients using PEG for bowel preparation. ${ }^{[8]}$

CONCLUSION: lectrolyte disturbance following PEG solutions are common but adverse reactions to it necesscitating ICU admission are rare. Increased awareness of this seemingly distinct and rare complication can help the treating physician to manage this entity effectively.

Our patient had presented with angioedema.

Anaphylaxis is a life-threatening reaction caused by the release of chemicals, such as histamine, leukotrienes and tryptase, from mast cells. Anaphylaxis is likely when all of the following 3 criteria are met:

- Sudden onset and rapid progression of symptoms

- Life-threatening Airway and/or Breathing and/or Circulation problems

- Skin and/or mucosal changes (flushing, urticaria, angioedema).[9]

Our patient had all these three symptoms. She was treated immediately with chlorpheniramine and hydrocortisone injection. Patient had complete resolution of symptoms.

The severity of presentation, need for excessive epinephrine requirement may prompt prolonged period of observation. Prompt recognition of anaphylaxis and its management is very essential.
Since the patient was kept on 24 hour fasting before surgery PEG solution should have been the cause for anaphylaxis. Previously only few cases had been reported and this case is being reported stressing on the importance of rare complication of a commonly used medication.

\section{REFERENCES:}

1. Assal A, Watson PY: Angioedema as a hypersensitivity reaction to polyethylene glycol oral electrolyte solution. Gastrointest Endosc 2006; 64: 294 -5.

2. Stollman N., and Manten H.D.: Angioedema from oral polyethylene glycol electrolyte lavage solution. Gastrointest Endosc 1996; 44: pp. 209-210.

3. Schuman E., and Balsam P.E.: Probable anaphylactic reaction to polyethylene glycol electrolyte lavage solution. Gastrointest Endosc 1991; 37: pp. 411.

4. Brullet E, Moron A, Calvet X, Frias C, Sola J: Urticarial reaction to oral polyethylene glycol electrolyte lavage solution. Gastrointest Endosc 1992; 38: 400 - 1.

5. Haneda T, et al. A case of anaphylaxis to Niflec. Skin Research 10: 199-202, No. 3, Jun 2011 PEG 4000/electrolytes S Anaphylaxis: case report.

6. Savitz, J. A., M.C.U.S.N., \& Durning, S. J., M.D. (2011). A rare case of anaphylaxis to bowel prep: A case report and review of the literature. Military Medicine, 176(8), 944-5.

7. Stefano Pizzimenti MD, Enrico Heffler MD, Elena Gentilcore MD, Alberto Raie MD, Claudia Bussolino MD, Franco NebioloMD and Giovanni Rolla MD.

8. Belsey, j., epstein, O. and Heresbach, D. (2009), Systematic review: adverse event reports for oral sodium phosphate and polyethylene glycol. Alimentary Pharmacology \& Therapeutics, 29: 15-28. Doi: 10.1111/j.13652036.2008.03837.

9. Annotated with links to NICE guidance July 2012 Review Date: 2016 Published by the Resuscitation Council (UK) 5th Floor, Tavistock House North Tavistock Square London WC1H 9HR. 